08

\title{
Гигантский магниторезистивный эффект в отражении микроволн от сверхрешеток (CoFe)/Cu
}

\author{
(ㄱ А.Б. Ринкевич, Е.А. Кузнецов, Д.В. Перов, М.А. Миляев \\ Институт фризики металлов им. М.Н. Михеева УрО РАН, \\ 620108 Екатеринбург, Россия \\ e-mail: rin@imp.uran.ru
}

Поступило в Редакцию 21 июля 2020 г.

В окончательной редакции 18 августа 2020 г.

Принято к публикации 19 августа 2020 г.

В интервале частот от 26 до $38 \mathrm{GHz}$ выполнено экспериментальное исследование микроволнового гигантского магниторезистивного эффекта в отражении волн. Эксперименты выполнены на сверхрешетках системы $(\mathrm{CoFe}) / \mathrm{Cu}$, которые имеют гигантское магнитосопротивление. Установлена величина эффекта (до $+3 \%$ в максимуме) и определена его зависимость от магнитного поля. Проведены расчеты полевой зависимости коэффициента отражения микроволн от магнитного поля. Измеренные значения изменения коэффициента отражения оказались больше рассчитанных. Различие связывается с приближением, в котором сверхрешетка в расчете заменяется однородной пластиной. Наблюдалась частотная зависимость микроволнового гигантского магниторезистивного эффекта в отражении, которая объяснена влиянием импеданса волновода, в который образец помещается при измерениях.

Ключевые слова: магнитные сверхрешетки, гигантский магниторезистивный эффект, микроволны, коэффициент отражения.

DOI: $10.21883 /$ JTF.2021.02.50367.229-20

\section{Введение}

Исследование микроволновых характеристик магнитных металлических сверхрешеток имеет как научное, так и практическое значение. В работе [1] было осуществлено первое наблюдение микроволнового гигантского магниторезистивного эффекта ( $\mu \mathrm{GMR})$ в отражении микроволн. Было установлено, что тип полевой зависимости $\mu \mathrm{GMR}$ в отражении волн по форме подобен эффекту в прохождении микроволн через сверхрешетку, однако $\mu \mathrm{GMR}$ в отражении имеет противоположный знак и значительно меньшую величину [2]. Различные условия реализации эффекта $\mu \mathrm{GMR}$ в широком интервале частот рассмотрены в работе [3], где из общего выражения для коэффициента прохождения электромагнитных волн через металлическую пластину были выбраны и рассмотрены два частных случая. Первый реализуется у металлических наноструктур с толщиной более единиц нанометров в микроволновом диапазоне длин волн. В этом случае в прохождении микроволн должно выполняться взаимно-однозначное соответствие $\mu \mathrm{GMR}$ и гигантского магниторезистивного эффекта (GMR), измеренного на постоянном токе. Взаимно-однозначное соответствие $\mu \mathrm{GMR}$ и GMR означает, что частотная зависимость $\mu \mathrm{GMR}$ должна отсутствовать. Второй предельный случай относится к наноструктурам с общей толщиной металла не более единиц нанометров. В этом частном случае взаимно-однозначное соответствие отсутствует.

Частотная зависимость $\mu \mathrm{GMR}$ подробно исследована в [4], где было установлено, что существуют причины, вызывающие зависимость $\mu \mathrm{GMR}$ от толщины нано- структуры и частоты волны. Расчеты коэффициентов прохождения и отражения, выполненные в настоящей работе, показали, что второй предельный случай не может реализоваться в металлических наноструктурах с толщинами более $0.5 \mathrm{~nm}$. Для волн сантиметрового и миллиметрового диапазонов в металлических наноструктурах с толщинами от $\sim 1.5$ до $\sim 200 \mathrm{~nm}$ реализуется первый предельный случай. Выполнение взаимнооднозначного соответствия $\mu \mathrm{GMR}$ и GMR неоднократно проверялось экспериментально [3-6]. Как правило, величина изменений коэффициента прохождения, измеренная в сантиметровом и миллиметровом диапазонах длин волн, хорошо коррелирует с магнитосопротивлением образцов. Частотная зависимость $\mu \mathrm{GMR}$ в прохождении микроволн для большинства образцов сверхрешеток $\mathrm{Fe} / \mathrm{Cr}$ выражена слабо. Лишь на некоторых образцах наблюдается некоторое уменьшение микроволнового магниторезистивного эффекта с ростом частоты [3]. Отличие от взаимно-однозначного соответствия $\mu \mathrm{GMR}$ и GMR наблюдается в инфракрасном диапазоне [7], а также на микроволнах для гранулярных систем [5].

Наноструктуры и сверхрешетки систем $\mathrm{Co} / \mathrm{Cu}$ и $(\mathrm{CoFe}) / \mathrm{Cu}$ обладают высоким магнитосопротивлением. $\mu \mathrm{GMR}$ в наноструктурах $\mathrm{Co} / \mathrm{Cu}$ и $(\mathrm{CoFe}) / \mathrm{Cu}$ изучался в работах $[8,9]$. В системе $\mathrm{Co} / \mathrm{Cu}$ наблюдалась слабая частотная зависимость $\mu \mathrm{GMR}$, причем с увеличением частоты $\mu \mathrm{GMR}$ уменьшается [8]. У сверхрешеток системы $(\mathrm{CoFe}) / \mathrm{Cu}$ зафиксировано рекордное на сегодняшний день микроволновое магнитосопротивление до $-80 \%$ [9]. Столь высокое магнитосопротивление обусловлено выбором сплава $\mathrm{Co}_{0.88} \mathrm{Fe}_{0.12}$, обладающего высоким GMR, выбором материала буферного слоя и 

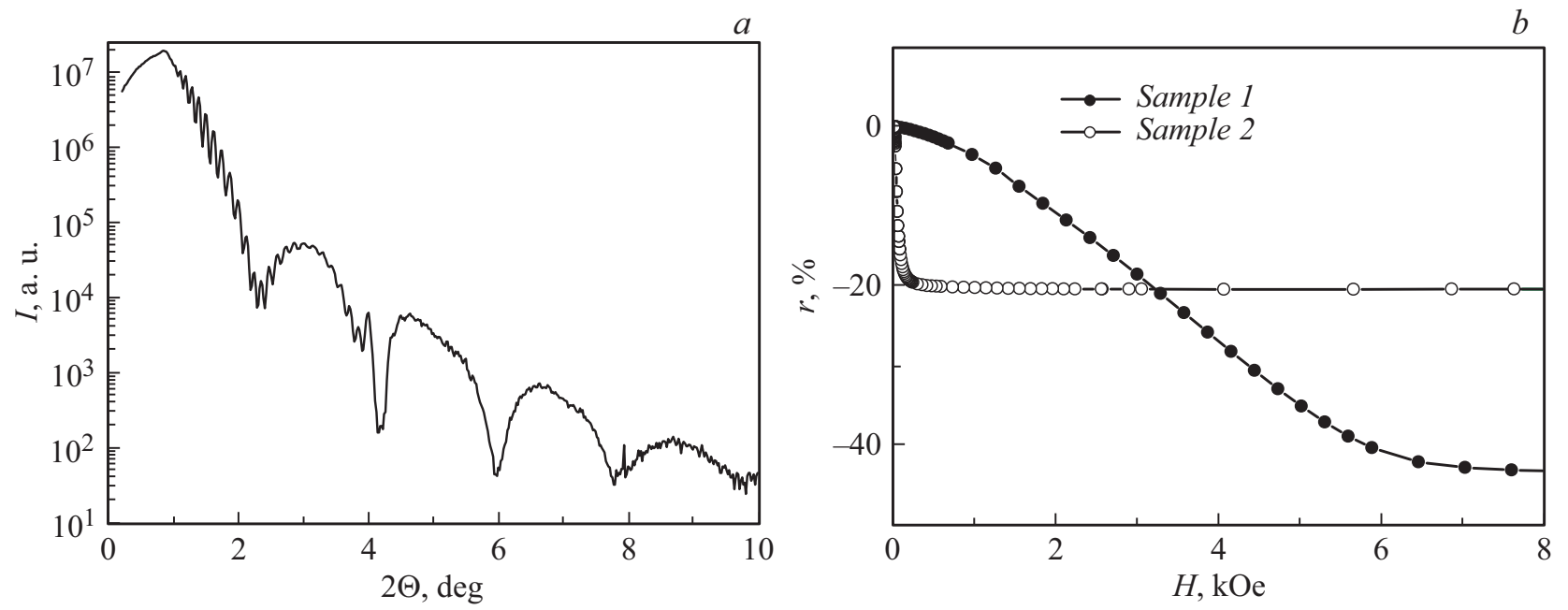

Рис. 1. Рентгеновская дифрактограмма для сверхрешетки $\mathrm{Ta}(5.0) / \operatorname{PyCr}(5.0) /\left[\mathrm{Co}_{88} \mathrm{Fe}_{12}(1.5) / \mathrm{Cu}(0.95)\right]_{24} / \mathrm{Ta}(5.0)$ (образец № 1) (a); изменение проводимости сверхрешеток в магнитном поле $(b)$.

оптимизацией технологии приготовления, что обеспечило требуемую микроструктуру образцов. В работе [6] выполнено наблюдение и проведен количественный анализ $\mu \mathrm{GMR}$ в спиновых клапанах, содержащих слои $(\mathrm{CoFe})$, обменно-связанные через прослойку (спейсер) Сu. Измерение отражения микроволн от спиновых клапанов, содержащих слои Со и спейсер $\mathrm{Cu}$, проведено модуляционным методом в [10].

Целью наших исследований является изучение $\mu \mathrm{GMR}$ в отражении микроволн для сверхрешеток $(\mathrm{CoFe}) / \mathrm{Cu}$, установление частотной зависимости $\mu \mathrm{GMR}$ в отражении и выяснение причины этой зависимости, а также осуществление совместного наблюдения FMR и $\mu \mathrm{GMR}$ в отражении микроволн. В настоящей работе исследованы микроволновые свойства двух сверхрешеток $\left[\left(\mathrm{Co}_{0.88} \mathrm{Fe}_{0.12}\right) / \mathrm{Cu}\right]_{n}$ с толщинами спейсера $t_{s}=0.95$ и $2.05 \mathrm{~nm}$. Такие толщины соответствуют первому и второму максимумам осцилляционной зависимости GMR от толщины спейсера. В этих образцах изменения сопротивления осуществляются в магнитных полях разной величины. Микроволновые измерения выполнены в интервале частот от 26 до $38 \mathrm{GHz}$. Измерены полевые зависимости коэффициента отражения.

\section{1. Образцы сверхрешеток и их аттестация}

Сверхрешетки $\left[\left(\mathrm{Co}_{0.88} \mathrm{Fe}_{0.12}\right) / \mathrm{Cu}\right]_{n}$ приготовлены методом магнетронного напыления с использованием установки MPS-4000-С6. Были приготовлены образцы сверхрешеток составов

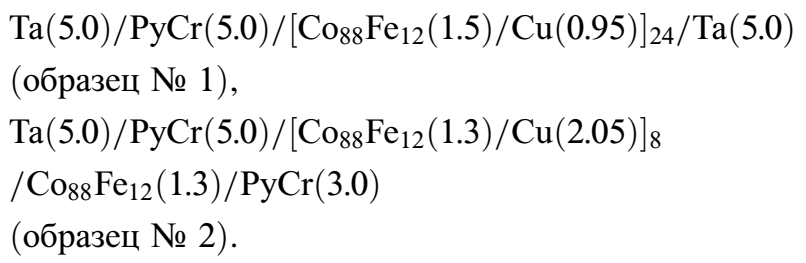

Число в круглых скобках указывает толщину данного слоя в нанометрах, индекс у квадратных скобок обозначает число пар слоев. Символом Ру обозначен сплав $\mathrm{Fe}-\mathrm{Ni}$. Толщина спейсера $\mathrm{Cu}$ выбрана таким образом, чтобы первый образец попадал на первый максимум зависимости величины GMR от толщины спейсера, a второй образец - на второй максимум. Образцы выращены на подложке из стекла Corning с толщиной $0.2 \mathrm{~mm}$. Технология роста сверхрешеток $(\mathrm{CoFe}) / \mathrm{Cu}$ описана в работе [11]. Буферный слой $\mathrm{Ta}(5.0)$, прилегающий к подложке, обеспечивает снятие внутренних напряжений в наноструктуре и формирует структуру, оптимальную для высокого магнитосопротивления. Верхний слой $\mathrm{Ta}(5.0)$ в образце № 1 или $\operatorname{PyCr}(3.0)$ в образце № 2 предохраняет наноструктуру от коррозии. Рентгеновское исследование образцов было выполнено в ЦКП ИФМ УрО РАН на дифрактометре PanAnalytical.

Для обеих сверхрешеток на дифрактограмме виден только один пик - от семейства плоскостей (111) ГЦК решетки. Этот пик - общий для $\mathrm{Cu}$ и сплава $\mathrm{Co}_{88} \mathrm{Fe}_{12}$ из-за того, что параметры ГЦК решетки меди и этого сплава очень близки. Вокруг данного пика имеются осцилляции. Их присутствие указывает на высокое совершенство слоистой структуры сверхрешеток (рис. 1, $a$ ). Вычисленный по угловому положению этих осцилляций период сверхрешеток практически совпадает с номинальными значениями. Отсутствие на дифрактограмме остальных характерных пиков ГЦК решетки указывает на то, что в данных образцах сформирована аксиальная текстура $\langle 111\rangle$, ось которой перпендикулярна плоскости пленки, ориентация которой подтверждена методом кривых качания. Совершенная текстура $\langle 111\rangle$ есть в обеих исследуемых сверхрешетках.

Рельеф поверхности образцов исследован методом атомно-силовой микроскопии с применением автоматического сканирующего зондового микроскопа Солвер Некст. Исследование показало, что образцы имеют глад- 

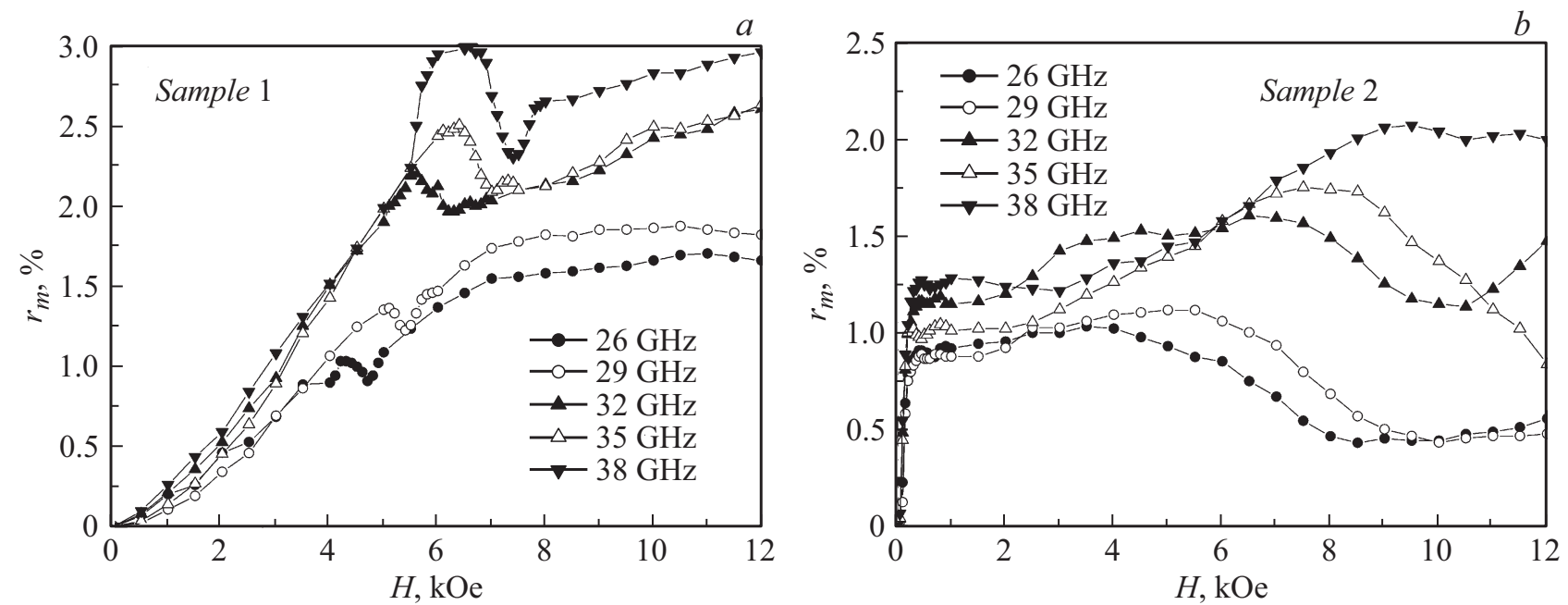

Рис. 2. Зависимости коэффициента отражения от магнитного поля, измеренные на нескольких частотах для сверхрешеток $\mathrm{Ta}(5.0) / \mathrm{PyCr}(5.0) /\left[\mathrm{Co}_{88} \mathrm{Fe}_{12}(15) / \mathrm{Cu}(0.95)\right]_{24} / \mathrm{Ta}(5.0)(a) ; \mathrm{Ta}(5.0) / \mathrm{PyCr}(5.0) /\left[\mathrm{Co}_{88} \mathrm{Fe}_{12}(1.3) / \mathrm{Cu}(2.05)\right]_{8} / \mathrm{Co}_{88} \mathrm{Fe}_{12}(1.3) / \mathrm{PyCr}(3.0)(b)$.

кую поверхность с разностью высот около $3 \mathrm{~nm}$ на поле сканирования $1 \times 1 \mu \mathrm{m}$.

Магнитные измерения показали, что образец № 1 имеет намагниченность насыщения $M_{s}=1625 \pm 8 \mathrm{Gs}$, образец № $2-1638 \pm 8$ Gs. Эти величины будут использованы ниже при выполнении расчетов. Для анализа результатов измерений $\mu \mathrm{GMR}$ требуется знание магнитосопротивления образца. Как известно, высокое магнитосопротивление реализуется в наноструктурах $(\mathrm{CoFe}) / \mathrm{Cu}$, если при отсутствии внешнего магнитного поля магнитные моменты слоев CoFe антипараллельны [10]. Магнитосопротивление измерено по четырехточечной схеме, результаты приведены на рис. $1, b$. Зависимость сопротивления для образца № 1, толщина спейсера которого соответствует первому максимуму GMR, имеет поле насыщения около $7 \mathrm{kOe}$. Значение магнитосопротивления $r=[\mathscr{R}(H)-\mathscr{R}(0)] / \mathscr{R}(0)$, где $\mathscr{R}(H)$ - электросопротивление образца в магнитном поле $H$ для этого образца в насыщении $r \approx-44 \%$. Зависимость магнитосопротивления образца № 2 насыщается в поле около $0.2 \mathrm{kOe}$, и величина магнитосопротивления в насыщении $r \approx-22 \%$. Толщина спейсера у образца № 2 соответствует второму максимуму GMR. Результаты измерений магнитосопротивления образцов согласуются с данными работы [11].

\section{2. Результаты микроволновых измерений}

Микроволновые измерения выполнены по методу, изложенному в работе [2]. Образец помещается в стандартный прямоугольный волновод сечением $3.6 \times 7.2 \mathrm{~mm}$ таким образом, что полностью перекрывает сечение волновода. С помощью измерителя амплитудно-частотных характеристик измеряется модуль коэффициента отражения $R$ и его изменение $r_{m}(H)$ в магнитном поле $H$ :
$r_{m}=[|R(H)|-|R(0)|] /|R(0)|$. При измерениях внешнее магнитное поле лежит в плоскости образца и направлено вдоль узкой стенки волновода. Таким образом, постоянное и микроволновое магнитные поля перпендикулярны. Измерения выполнены при комнатной температуре.

Измерения зависимости модуля коэффициента отражения от магнитного поля выполнены на нескольких частотах миллиметрового диапазона. Результаты для образца № 1 представлены на рис. 2, а. Эти изменения обусловлены действием двух причин. Первая - это изменение сопротивления сверхрешетки в магнитном поле. Эта причина приводит к монотонному увеличению коэффициента отражения и выражает эффект $\mu \mathrm{GMR}$. Вторая вызвана ферромагнитным резонансом (FMR). Она проявляется в относительно узкой области магнитных полей и, как правило, приводит к уменьшению коэффициента отражения из-за поглощения микроволн при резонансе. Как видно из рис. 2, $a$, поле резонанса увеличивается с ростом частоты. Оценки по формулам для спектра резонанса, приведенные в [12], показали, что поля, где происходят изменения резонансного типа, соответствуют спектру однородного FMR. На частотах 35 и $38 \mathrm{GHz}$ наблюдается более сложная картина явлений. B полях несколько ниже поля FMR происходит увеличение коэффициента отражения. Такого явления ранее не наблюдалось при изучении отражения микроволн от сверхрешеток $\mathrm{Fe} / \mathrm{Cr}[2]$ и спин-вентильных структур $(\mathrm{CoFe}) / \mathrm{Cu}[9]$. Для выяснения причины этого увеличения коэффициента отражения нужны дополнительные исследования. В качестве предположения можно рассмотреть возможность реализации магнитного антирезонанса [12]. В пользу этого предположения говорят следующие обстоятельства: особенность проявляется в виде максимума коэффициента отражения, который реализуется в полях, меньших поля FMR; эта особенность сильно проявляется на частотах, превышающих $32 \mathrm{GHz}$. Такие проявления должны быть при антирезонансе. 
Вернемся к анализу $\mu \mathrm{GMR}$ в первом образце. Из рис. 2, a видно, что с ростом частоты величина изменений коэффициента в сильных полях в целом увеличивается. Этот факт не согласуется с теорией эффекта $\mu \mathrm{GMR}[2,4]$. Причина этого несоответствия будет обсуждаться в следующем разделе. Во втором образце магнитное насыщение осуществляется в полях $0.3 \mathrm{kOe}$, поэтому области $\mu \mathrm{GMR}$ и FMR разделены (рис. 2,b). $\mathrm{B}$ полях до $2 \mathrm{kOe}$ наблюдается только $\mu \mathrm{GMR}$. Здесь также можно различить частотную зависимость этого эффекта, причем с увеличением частоты величина изменений коэффициента отражения возрастает. В более сильных полях наблюдаются изменения коэффициента, вызванные очень широкой линией FMR. Отметим, что и для этого образца на частотах 32 и $35 \mathrm{GHz}$ в полях, меньших поля FMR, наблюдается увеличение коэффициента отражения, которое может быть вызвано магнитным антирезонансом.

\section{Обсуждение}

Расчету коэффициентов прохождения микроволн через пластину металла и отражения от нее посвящено несколько работ, см., например, $[13,14]$. Этот расчет был применен к анализу $\mu \mathrm{GMR}[6]$. В указанных работах коэффициенты прохождения и отражения вводятся как отношения мощностей прошедшей и отраженной волн к мощности падающей волны. В этом варианте для выполнения расчетов требуется знание поверхностного сопротивления образца. В используемом нами варианте расчета коэффициент отражения вводится как отношение амплитуд отраженной и падающей волн. Здесь знание поверхностного сопротивления не требуется. Оба варианта расчета основаны на приближении, в котором многослойная наноструктура заменяется пластиной однородного материала с такой же проводимостью и такой же магнитной проницаемостью. Расчет здесь выполнен подобно работе [2]. Коэффициент отражения $R$ зависит от соотношения импедансов наноструктуры $Z_{m}$ и окружающей среды $Z$ (это импеданс волновода либо импеданс свободного пространства), а также от соотношения толщины металла наноструктуры $d$ и глубины скин-слоя $\delta$. В условиях наших экспериментов реализуется неравенство $d \ll \delta$. Для нормального скинэффекта импеданс металлической наноструктуры равен $Z_{m}=[(1+i) / \delta] \rho$, где $\rho=\rho(H)-$ удельное электросопротивление наноструктуры, $\delta=\left(2 \rho / \omega \mu \mu_{0}\right)^{1 / 2}-$ глубина скин-слоя, $\omega=2 \pi f-$ круговая частота, $\mu-$ относительная динамическая дифференциальная магнитная проницаемость. Здесь и далее под магнитной проницаемостью и электросопротивлением понимаются эффективные величины, усредненные по объему сверхрешетки. Импеданс волновода, в который помещена наноструктуpa, на волне типа $\mathrm{TE}_{10}$ рассчитывается по формуле

$$
Z=\left(\mu_{0} / \varepsilon_{0}\right)^{1 / 2} /\left[\left(1-\left(\lambda / \lambda_{c}\right)^{2}\right]^{1 / 2},\right.
$$

где $\lambda=c / f$ - длина волны в вакууме, $\lambda_{c}=2 a-$ критическая длина волны моды $\mathrm{TE}_{10}, a$ - ширина большей стенки прямоугольного волновода. В наших экспериментах $a=7.2 \mathrm{~mm}$. Если измерения выполняются не в волноводе, а в свободном пространстве, то вместо (1а) импеданс определяется по формуле (1б)

$$
Z=\left(\mu_{0} / \varepsilon_{0}\right)^{1 / 2}=522 \Omega \text {. }
$$

Согласно [15], формулу для расчета коэффициента отражения $R$ электромагнитной волны можно записать так

$$
R=-1+\frac{2 Z_{m} \operatorname{ch}\left(k_{m} d\right)}{2 Z_{m} \operatorname{ch}\left(k_{m} d\right)+Z \operatorname{sh}\left(k_{m} d\right)},
$$

где $k_{m}-$ волновое число в проводящей среде, $k_{m}=(1+i) / \delta$. Импеданс хорошо проводящей наноструктуры намного меньше импеданса $Z,\left|Z_{m}\right| \ll Z$. Если в знаменателе (2) имеет место неравенство $2 Z_{m} \operatorname{ch}\left(k_{m} d\right) \ll Z \operatorname{sh}\left(k_{m} d\right)$, то коэффициент отражения можно найти по формуле

$$
R=-1+\frac{2 Z_{m}}{Z} \operatorname{cth}\left(k_{m} d\right)
$$

Этот случай реализуется на микроволновых частотах для металлических наноструктур. В этом предельном случае коэффициент $R$ может зависеть от частоты изза частотной дисперсии материальных констант и из-за частотной зависимости импеданса волновода $Z$. Эта зависимость импеданса $Z$ слаба вдали от частоты отсечки волновода $f_{c}=\frac{c}{2 a}$, но она может сказаться, если нет других источников дисперсии. Из (3) для области параметров, когда можно пренебречь изменением магнитной проницаемости, можно получить для относительного изменения коэффициента отражения

$$
r_{m}=\frac{|R(H)|-|R(0)|}{|R(0)|}=-\frac{2 \rho(0) r}{Z d \mu(0)}\left(1-\frac{2 \rho(0)}{Z d \mu(0)}\right) .
$$

В (4) $\rho(0)$ - удельное сопротивление и $\mu(0)-$ магнитная проницаемость в нулевом магнитном поле. Величина $D(0)=\frac{2 \rho(0)}{Z d \mu(0)}$ для металлических пленок толщиной более $10 \mathrm{~nm}$ много меньше единицы, $D(0) \ll 1$. Если изменения коэффициента отражения вызваны только магнитосопротивлением наноструктуры при $\mu(0) \approx 1$, то из (4) получается

$$
r_{m}=-D(0)[1-D(0)] r .
$$

Отсюда следует, что изменения коэффициента отражения имеют знак, противоположный знаку магнитосопротивления $r$. Изменения $r_{m}$ существенно меньше $|r|$ по величине, но идентичны $r$ по характеру зависимости от напряженности внешнего магнитного поля. Формулы (4) и (5) справедливы, если наноструктура находится вдали от условия ферромагнитного резонанса.

Проведем расчет величины коэффициента отражения для сверхрешеток, исследованных в настоящей работе. Результаты расчетов по формулам (5), (1a), (1б) приведены в таблице. Расчет выполнен для частоты волны $f=35 \mathrm{GHz}$ в магнитном поле $12 \mathrm{kOe}$, в котором образец 

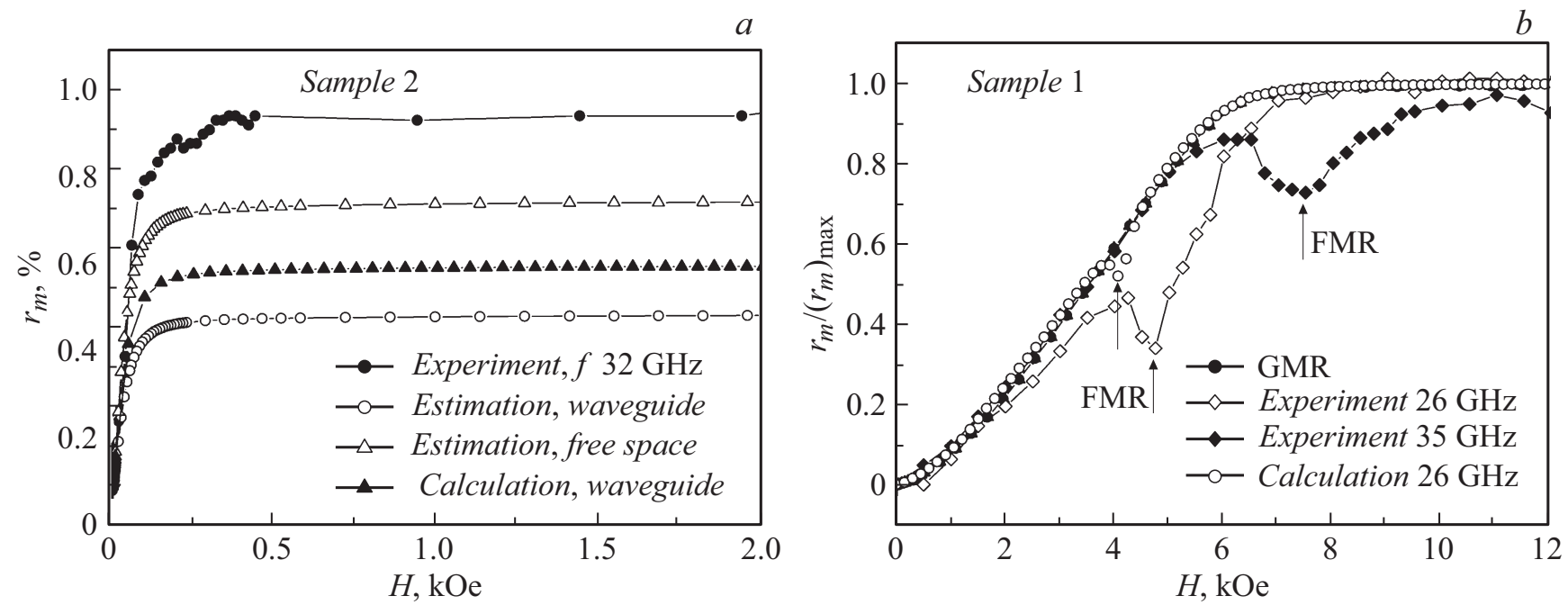

Рис. 3. Сопоставление измеренных и рассчитанных полевых зависимостей микроволнового коэффициента отражения для сверхрешетки $\mathrm{Ta}(5.0) / \mathrm{PyCr}(5.0) /\left[\mathrm{Co}_{88} \mathrm{Fe}_{12}(1.3) / \mathrm{Cu}(2.05)\right]_{8} / \mathrm{Co}_{88} \mathrm{Fe}_{12}(1.3) / \mathrm{PyCr}(3.0)(a)$; сопоставление формы полевой зависимости $\mu \mathrm{GMR}$ и GMR для сверхрешетки $\mathrm{Ta}(5.0) / \mathrm{PyCr}(5.0) /\left[\mathrm{Co}_{88} \mathrm{Fe}_{12}(1.5) / \mathrm{Cu}(0.95)\right]_{24} / \mathrm{Ta}(5.0)(b)$.

Сопоставление экспериментального и расчетного изменения коэффициента отражения

\begin{tabular}{l|c|c|c|c|c}
\hline \multirow{2}{*}{$\begin{array}{l}\text { Номер } \\
\text { образца }\end{array}$} & $\begin{array}{c}\text { Полная } \\
\text { толщина } \\
\text { металла, } \\
\text { nm }\end{array}$ & $\begin{array}{c}\text { Удельная } \\
\text { электро- } \\
\text { проводность, } \\
10^{-6} \Omega \cdot \mathrm{m}\end{array}$ & \multicolumn{2}{|c|}{ Относительное изменение коэффициента отражения } \\
\cline { 4 - 6 } & 71.8 & 0.483 & 2.6 & $\begin{array}{c}\text { расчет для } \\
\text { волновода, \% }\end{array}$ & $\begin{array}{c}\text { расчет для свободного } \\
\text { пространства, \% }\end{array}$ \\
\hline 1 & 41.1 & 0.283 & 1.0 & 1.5 & 1.71 \\
2 & & & 0.62 & 0.71
\end{tabular}

№ 1 находится в состоянии, близком к магнитному насыщению, и в поле $2 \mathrm{kOe,} \mathrm{в} \mathrm{котором} \mathrm{образец} \mathrm{№} 2$ находится в состоянии насыщения. Из таблицы видно, что экспериментально измеренное изменение коэффициента отражения для обеих сверхрешеток превышает рассчитанное на 50-60\%. На рис. 3, а показаны экспериментально измеренная зависимость коэффициента отражения для образца № 2, а также оценки коэффициента, рассчитанные по формуле (5) для импеданса, рассчитанного для волновода по формуле (1) и для свободного пространства по формуле (1б). Кроме этого, выполнен расчет коэффициента отражения по формуле (2), которая содержит меньше приближений. Мы видим соответствие типа всех зависимостей, но отличие в величине изменений. Экспериментально измеренные величины изменений оказались больше рассчитанных. По-видимому, это различие, в первую очередь между экспериментом и расчетом по формуле (2), связано с приближениями, принятыми при замене многослойной сверхрешетки однородной пластиной с эквивалентной проводимостью. Отметим, что в сверхрешетках $(\mathrm{CoFe}) / \mathrm{Cu}$ наблюдалось превышение экспериментально измеренного $\mu \mathrm{GMR}$ в прохождении микроволн над рассчитанным в том же приближении [16].

Проведем сопоставление формы полевой зависимости $\mu \mathrm{GMR}$ в отражении и GMR. Сопоставление проведем для образца № 1. По сравнению с образцом № 2 это более сложный случай, так как на полевых зависимостях кроме $\mu \mathrm{GMR}$ присутствуют еще изменения, вызванные FMR. Результат сопоставления показан на рис. 3, b, где по оси $Y$ отложена величина $r_{m}$, нормированная на свое максимальное значение в насыщении, а именно $r_{m} /\left(r_{m}\right)_{\max }$. На рис. $3, b$ проведено сопоставление GMR, взятого с обратным знаком, и полевой зависимости коэффициента отражения, измеренной на двух частотах 26 и $35 \mathrm{GHz}$. Видно, что кроме областей, близких к условию FMR, форма зависимостей GMR и $\mu \mathrm{GMR}$ практически идентична. Вблизи условия FMR величина коэффициента отражения уменьшается из-за поглощения волны [2]. На рис. 3,b положение FMR отмечено стрелками. Монотонная зависимость коэффициента отражения, вызванная $\mu \mathrm{GMR}$, почти полностью повторяет зависимость GMR. Результаты расчета по оценочной формуле (5) точно совпадают с зависимостью нормированного магнитосопротивления при GMR, и на рис. $3, b$ они не показаны.

Заслуживают обсуждения причины частотной зависимости коэффициента отражения, зафиксированной на рис. 2. Для образца № 1 эта зависимость ясно видна в интервале полей 1-12 kOe, а для образца № 2 около $2 \mathrm{kOe}$. В этом поле образец № 2 находится в магнитном насыщении, а условие ФМР осуществляется 


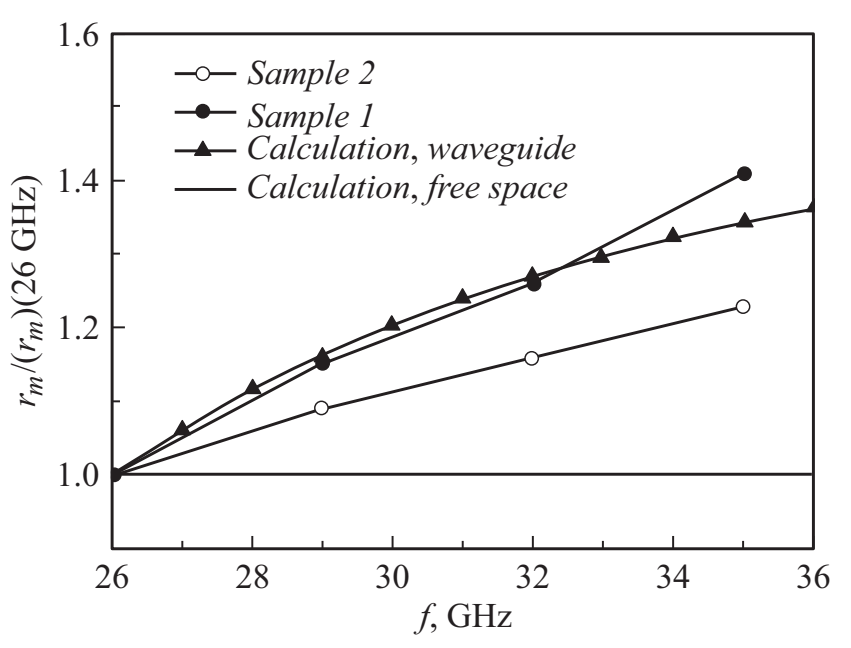

Рис. 4. Частотная зависимость изменений коэффициента отражения при $\mu \mathrm{GMR}$, нормированная на коэффициент отражения на частоте $f=26 \mathrm{GHz}$.

в значительно более сильных полях. В обоих случаях прослеживается тенденция - с увеличением частоты волны величина эффекта $\mu \mathrm{GMR}$ возрастает. Упрощенная формула (5) не предполагает частотной зависимости $\mu \mathrm{GMR}$, если нет частотной зависимости электросопротивления. При следующем из величины электросопротивления времени релаксации $\tau \sim 10^{-14}-10^{-15}$ s временная дисперсия на наших частотах $f \sim 3 \cdot 10^{10} \mathrm{~Hz}$ не может осуществляться, так как $2 \pi f \tau \ll 1$. Возможность частотной зависимости проводимости тонкой металлической пластины предполагается в работе [17] за счет пространственной, а не временной дисперсии проводимости. Эта точка зрения была, однако, подвергнута критике, и сделано заключение об отсутствии частотной зависимости проводимости в интервалах миллиметровых и субмиллиметровых волн [18]. В работе [4] показано, что на частотах верхней части миллиметрового диапазона следует учитывать возможность установления стоячих волн в диэлектрической подложке, что приводит к частотной зависимости коэффициентов отражения при $\mu \mathrm{GMR}$. Использованные в настоящей работе частоты значительно ниже, и возможности установления стоячих волн нет. В принципе, частотная зависимость коэффициента отражения содержится в формулах (2) и (5), если импеданс волновода $Z$ рассчитывается по формуле (1a). Выполним оценки влияния частотной зависимости импеданса волновода. Изменения коэффициента отражения, произошедшие из-за $\mu \mathrm{GMR} r_{m}$ для каждого образца, нормируем на изменения в нем на низшей частоте $26 \mathrm{GHz}: r_{m} /\left(r_{m}\right)(26 \mathrm{GHz})$. Результаты представлены на рис. 4. Как уже отмечалось, с увеличением частоты величина изменений возрастает. На рис. 4 показана также рассчитанная по формуле (2) зависимость нормированных изменений коэффициента отражения, если импеданс рассчитан по формуле (1) для волновода. Импеданс свободного пространства частотно независим, так что в этом случае частотной зависимости коэффициента отражения нет. Из сравнения экспериментальных данных и расчета на рис. 4 можно сделать заключение, что для образца № 1 получено хорошее согласие эксперимента и расчета, а для образца № 2 согласие по порядку величины. В целом можно утверждать, что частотная зависимость изменений коэффициента отражения при $\mu \mathrm{GMR}$ в наших условиях в значительной степени вызвана частотной зависимостью импеданса волновода.

\section{Заключение}

Исследован микроволновой гигантский магниторезистивный эффект в отражении для двух образцов сверхрешеток $\left(\mathrm{Co}_{88} \mathrm{Fe}_{12}\right) / \mathrm{Cu}$ с толщинами спейсера $\mathrm{Cu}$, соответствующими первому и второму максимуму зависимости эффекта GMR от толщины спейсера. Установлено, что в полевой зависимости коэффициента отражения на микроволновых частотах присутствует резонансный вклад, вызванный FMR. Установлено, что вызванные $\mu \mathrm{GMR}$ изменения коэффициента отражения имеют тенденцию к насыщению в полях, соответствующих магнитному насыщению образца. Величина относительных изменений коэффициента отражения при $\mu \mathrm{GMR}$ достигает величины от 1 до 4\%. Изменения в соответствии с теорией имеют положительный знак и форму полевой зависимости, идентичную гигантскому магниторезистивному эффекту. Величина изменений в эксперименте превышает рассчитанные величины, и различие связывается с приближением в расчетах, когда многослойная сверхрешетка заменяется однородной пластиной с эффективной проводимостью. Экспериментально обнаружено, что изменения коэффициента при $\mu \mathrm{GMR}$ имеют частотную зависимость, которая может быть объяснена частотной зависимостью импеданса волновода, в который помещен образец при измерениях.

\section{Благодарности}

Авторы благодарны М.В. Макаровой, Е.А. Кравцову за выполнение рентгеновского исследования наноструктур в ЦКП ИФМ УрО РАН.

\section{Финансирование работы}

Работа выполнена в рамках темы „Спин“ № AАAАА18-118020290104-2 и „Функция“ № АAАА-А19119012990095-0. Разд. 2 выполнен при поддержке гранта РНФ № 17-12-01002.

\section{Конфликт интересов}

Авторы заявляют, что у них нет конфликта интересов. 


\section{Список литературы}

[1] Z. Frait, P. Sturć, K. Temst, Y. Bruynseraede, I. Vavra. Solid State Comm., 112, 569 (1999).

[2] В.В. Устинов, А.Б. Ринкевич, Л.Н. Ромашев, Е.А. Кузнецов. Письма в ЖТФ, 33 (18), 23 (2007).

[3] В.В. Устинов, А.Б. Ринкевич, Л.Н. Ромашев, А.М. Бурханов, Е.А. Кузнецов. ФММ, 96 (3), 52 (2003).

[4] Д.В. Перов, А.Б. Ринкевич. ФММ, 120 (4), 360 (2019).

[5] D.P. Belozorov, V.N. Derkach, S.V. Nedukh. Int. J. Infrared Millimeter Waves, 22 (11), 1669 (2001).

[6] D.E. Endean, J.N. Heyman, S. Maat, E. Dan Dahlberg. Phys. Rev. B, 84, 212405 (2011).

[7] J.C. Jackuet, T. Valet. Mater. Res. Soc. Symp. Proc. Magnetic Ultrathin Films, Multilayers and Surfaces, USA, San Francisco, Pittsburgh, Pennsylvania, 384, 477 (1995).

[8] T. Rausch, T. Szczurek, M. Schlesinger. J. Appl. Phys., 85 (1), 314 (1999).

[9] А.Б. Ринкевич, Я.А. Пахомов, Е.А. Кузнецов, А.С. Клепикова, М.А. Миляев, Л.И. Наумова, В.В. Устинов. Письма в ЖТФ, 45 (5), 42 (2019).

[10] J. Dubowik, F. Stobiecki, I. Gościańska. Czechoslovak J. Phys., 52 (2), 227 (2002).

[11] М.А. Миляев, Л.И. Наумова, В.В. Устинов. ФММ, 119 (12), 1 (2018).

[12] А.Г. Гуревич, Г.А. Мелков. Магнитные колебания и волны (Физматлит, М., 1994)

[13] R.L. Ramey, W.J. Kitchen, J.M. Lloyd, H.S. Landes. J. Appl. Phys., 39 (8), 3883 (1968).

[14] R.L. Ramey, T.S. Lewis. J. Appl. Phys., 39 (3), 1747 (1968).

[15] Н.А. Семенов. Техническая электродинамика (Связь, М., 1972).

[16] В.В. Устинов, А.Б. Ринкевич, И.Г. Важенина, М.А. Миляев. ЖЭТФ, 131, 140 (2020).

[17] W. Pan, X. Zhang. Int. J. Infrared Millim. Waves, 27 (3), 455 (2006).

[18] S. Lucyszyn. Int. J. Infrared Millim. Waves, 28, 263 (2007). 